\title{
Amine-Functionalized Zeolitic Imidazolate Framework-8 (ZIF-8) Nanocrystals for Adsorption of Radioactive Iodine
}

\footnotetext{
Yu Ri Lee ${ }^{\mathrm{a}, \dagger}$, Xuan Huy Do ${ }^{\mathrm{a}, \mathrm{b}, \dagger}$, Kie Yong Choc ${ }^{\mathrm{c}}$, Keunhong Jeong ${ }^{\mathrm{d}}$, and Kyung-Youl Baek ${ }^{\mathrm{a}, \mathrm{b}, \mathrm{e} *}$

a Materials Architecturing Research Center, Korea Institute of Science and Technology, Seoul 02792, Republic of Korea.

${ }^{b}$ Division of Nano \& Information Technology, KIST School, Korea University of Science and Technology, Seoul 02792, Republic of Korea.

${ }^{c}$ Department of Industrial Chemistry, Pukyong National University, Busan 608-739, Korea.

${ }^{d}$ Department of Chemistry, Nuclear \& WMD Protection Research Center, Korea Military Academy, Seoul, 01805, South Korea.

${ }^{e}$ Center for Convergent Chemical Process, Korea Research Institute of Chemical Technology, 141, Gajeong-ro, Yuseong-gu, Daejeon 34114, Republic of Korea.

${ }^{\dagger}$ These authors have equal contributions to this work.

*Corresponding author E-mail: baek@kist.re.kr
} 


\section{SI-1. Experimental details}

\subsection{Synthesis of ZIF-8}

ZIF-8 was synthesized on the basis of our recent report described by Cho et al. ${ }^{1}$ Typically, two separate solutions of $2.9 \mathrm{~g}$ of $\mathrm{Zn}\left(\mathrm{NO}_{3}\right) \cdot 6 \mathrm{H}_{2} \mathrm{O}$ and $6.9 \mathrm{~g}$ of 2-mIM were dissolved in $100 \mathrm{~mL}$ of $\mathrm{MeOH}$, respectively. Then, zinc salt solution was mixed with the 2-mIM solution under vigorous stirring for $2 \mathrm{~h}$ at $30{ }^{\circ} \mathrm{C}$. After the synthesis reaction, the white color precipitates were filtered by centrifugation, washed three times with $\mathrm{MeOH}$, and dried at $70{ }^{\circ} \mathrm{C}$ for $24 \mathrm{~h}$ to obtain the ZIF-8 product.

\subsection{Synthesis of amine-functionalized ZIF-8 (ZIF8-A) nanocrystal materials}

Amine-functionalized ZIF-8 was prepared by the ligand exchange of 2-mIM with Atz via post-synthetic modification method, according to the previous report. ${ }^{1} 200 \mathrm{mg}$ of the activated ZIF-8 well dispersed in $100 \mathrm{~mL}$ of $\mathrm{MeOH}$ using sonication for $30 \mathrm{~min}$ and then, 585 mg of Atz was added to the ZIF-8 suspension. The synthesis reaction was carried out for a certain reaction time at $50{ }^{\circ} \mathrm{C} ; 1 \mathrm{~h}, 8 \mathrm{~h}$, and $24 \mathrm{~h}$ for $15 \%, 37 \%$, and $61 \%$ of Atz conversion, respectively. After a certain time, the obtained products were separated, washed several times with $\mathrm{MeOH}$, and dried at $70{ }^{\circ} \mathrm{C}$ for 3 days under vacuum. The final products obtained were designated as ZIF8-Ax depending on the Atz conversion in ZIF-8 (e.g., ZIF8-A61 sample contains $61 \%$ of Atz conversion in ZIF-8).

\subsection{Characterization}

X-ray diffraction (XRD) analysis was performed on a Rigaku diffractometer (Rigaku Smart Lab, Rigaku Co., Japan) operated at $40 \mathrm{kV}$ and $40 \mathrm{~mA}$ using a diffracted beam monochromator with $\mathrm{Cu} K_{\alpha}(\lambda=1.54 \AA)$ radiation at a scan rate of $2^{\circ} / \mathrm{min} .{ }^{1} \mathrm{H}-\mathrm{NMR}$ spectra were measured in a mixture of $\mathrm{H}_{2} \mathrm{SO}_{4} / \mathrm{DMSO}-d_{6}(90 / 10, \mathrm{v} / \mathrm{v})$ on a Bruker Avance III $400 \mathrm{MHz}$ NMR 
spectrometer at $25^{\circ} \mathrm{C}$. The Brunauer-Emmett-Teller (BET) surface areas and pore volumes of all samples were determined from $\mathrm{N}_{2}$ adsorption-desorption isotherms using a BELsorp-mini instrument (BEL, Japan) at $77 \mathrm{~K}$. Before the adsorption measurements, the samples were pretreated at $150^{\circ} \mathrm{C}$ overnight under a vacuum. The morphology and shape of all the materials were characterized by field-emission scanning electron microscopy (FE-SEM, Inspect F50) and transmission electron microscopy (TEM, Tecnai F20 G2). Thermal stability of the materials was measured on thermogravimetric analysis (TGA) Q50, TA instrument. The TGA measurement was conducted by heating the samples from $50{ }^{\circ} \mathrm{C}$ to $700{ }^{\circ} \mathrm{C}$ at a heating rate of $10^{\circ} \mathrm{C} \mathrm{min}^{-1}$. Before TGA analysis, all the samples were pre-heated at $150{ }^{\circ} \mathrm{C}$ for $24 \mathrm{~h}$ to remove any residual solvents. X-ray photoelectron spectroscopy (XPS) profiles were recorded on a PHI 5000 VersaProbe (Ulvac-PHI, Japan) with a monochromated Al Ka (1486.6 eV) X-ray source. Raman spectra were obtained using a Renishaw Invia Raman microscope with a 782-nm laser. Fourier transform infrared (FT-IR) spectra were acquired on a Nicolet IS10 spectrometer (Thermo Scientific) after sample activation. The iodine concentrations of the initial and filtered solutions were obtained using UV-vis spectrophotometry (Jasco V-670) between 200 and 700 $\mathrm{nm}$. 


\section{SI-2. Iodine adsorption/desorption experiments}

2.1. Adsorption of iodine. An $I_{2}$ stock solution $(10000 \mathrm{mg} / \mathrm{L})$ in $n$-hexane was prepared and diluted to different concentrations for the adsorption experiments. The $\mathrm{I}_{2}$ concentrations were determined from ultraviolet (UV)-visible absorption measurements at $521 \mathrm{~nm}$ and the corresponding calibration curve. Before $\mathrm{I}_{2}$ adsorption, the ZIF-8 and ZIF8-A adsorbents were pretreated at $130{ }^{\circ} \mathrm{C}$ in a vacuum oven for $12 \mathrm{~h}$. After each adsorption run, the adsorbents were separated from the $I_{2}$ solution using a $0.22 \mu \mathrm{m}$ polyethylene terephthalate (PET) syringe filter. When the $\mathrm{I}_{2}$ adsorption experiment was conducted in n-hexane phase, no solvent reduction phenomenon occurred which was confirmed by comparing the amount of the solvent before and after adsorption. The equilibrium adsorption capacities $\left(q_{e}, \mathrm{mg} / \mathrm{g}\right)$ for $\mathrm{I}_{2}$ were calculated using eq 1.

$$
q_{e}=\left(C_{\mathrm{i}}-C_{\mathrm{e}}\right) \times V / m
$$

Here, $C_{\mathrm{i}}$ and $C_{\mathrm{e}}$ are the initial and equilibrium concentrations $(\mathrm{mg} / \mathrm{L})$ of $\mathrm{I}_{2}$ in the solution, respectively, $V$ is the volume of the solution (L), and $m$ is the weight of the adsorbent (g).

2.2. Adsorption kinetics. To analyze the $I_{2}$ adsorption kinetics on ZIF-8 and ZIF8-A, the pseudo-first-order (PFO) and pseudo-second-order (PSO) kinetic models ${ }^{2}$ were applied to fit the experimental data. The linear forms of these two models are expressed by eqs (2) and (3).

$$
\begin{gathered}
\log \left(q_{e}-q_{t}\right)=\log q_{e}-\left(k_{1} / 2.303\right) t \\
t / q_{t}=\left(1 / k_{2} q_{e}{ }^{2}\right)+\left(t / q_{t}\right)
\end{gathered}
$$

Here, $q_{e}$ and $q_{t}$ are the amounts of $\mathrm{I}_{2}$ adsorbed on adsorbent $(\mathrm{mg} / \mathrm{g})$ at equilibrium and time $t$, and $k_{1}\left(\mathrm{~h}^{-1}\right)$ and $k_{2}\left(\mathrm{~g} \mathrm{mg}^{-1} \mathrm{~h}^{-1}\right)$ are the rate constants of the PFO and PSO kinetic models, respectively. The intercept and slope of $\log \left(q_{e}-q_{t}\right)$ versus $t$ and $t / q_{t}$ versus $t$ were used to 
determine the values of $k_{1}$ and $k_{2}$, respectively. The initial adsorption rate, $h,\left(\mathrm{mg} \mathrm{g}^{-1} \mathrm{~min}^{-1}\right.$ ) and half equilibrium time, $t_{1 / 2}$, (h) can also be determined based on the PSO kinetic rate constant using eqs (4) and (5), respectively.

$$
\begin{aligned}
& h=k_{2} q_{e}{ }^{2} \\
& t_{1 / 2}=1 / k_{2} q_{e}
\end{aligned}
$$

2.3. Adsorption isotherms. The Langmuir isotherm model, which assumes that monolayer adsorption takes place over the adsorbent surface, is given by eq (6), ${ }^{3}$ whereas the Freundlich model, which assumes that adsorption processes occur on heterogeneous surfaces, ${ }^{4}$ is given by eq (7).

$$
\begin{gathered}
q_{e}=\frac{q_{m} K_{L} C_{e}}{1+K_{L} C_{e}} \\
q_{e}=K_{F} C_{e^{\frac{1}{n}}}^{\frac{1}{}}
\end{gathered}
$$

Here, $q_{e}(\mathrm{mg} / \mathrm{g})$ is the amount of $\mathrm{I}_{2}$ adsorbed per unit amount of adsorbent at equilibrium, $q_{m}$ (mg/g) is the maximum adsorption, and $C_{e}(\mathrm{mg} / \mathrm{L})$ is the equilibrium concentration of $\mathrm{I}_{2}$ in solution. $K_{L}$ and $K_{F}$ are the Langmuir constant, representing the adsorbate for the adsorption sites, and Freundlich constant, indicating adsorption capacity related to adsorbate-adsorbent interactions, respectively. Finally, $1 / n$ is the adsorption intensity, indicating the relative distribution of the energy and heterogeneity of the adsorbate sites.

2.4. Adsorption of Iodine vapor. $10 \mathrm{mg}$ of ZIFs was placed in open vial and $2 \mathrm{~g}$ of iodine was placed in another open vial. Both vials were put together in a chamber (Scheme S1). The chamber was evacuated using a vacuum pump for 30 min in room temperature (with $0 \% \mathrm{RH}$ ) and placed in a conventional oven which was preheated at $75^{\circ} \mathrm{C}$. Sample weights were checked at a certain time. 
Table S1. Kinetics parameters of the pseudo-first-order and pseudo-second-order models for $I_{2}$ adsorption on prepared adsorbents

\begin{tabular}{|c|c|c|c|c|c|c|c|c|c|}
\hline \multirow[t]{2}{*}{ Adsorbent } & \multicolumn{4}{|c|}{ Pseudo-first-order kinetics } & \multicolumn{5}{|c|}{ Pseudo-second-order kinetics } \\
\hline & $\begin{array}{c}q_{e, \exp } \\
\left(\mathrm{mg} \mathrm{g}^{-1}\right)\end{array}$ & $\begin{array}{c}q_{e, c a l} \\
\left(\underset{1}{\mathrm{mg} \mathrm{g}^{-}}\right)\end{array}$ & $\begin{array}{c}k_{1} \\
\left(\mathrm{~h}^{-1}\right)\end{array}$ & $R^{2}$ & $\begin{array}{c}q_{e, c a l} \\
\left(\underset{1}{\mathrm{mg} \mathrm{g}^{-}}\right)\end{array}$ & $\begin{array}{c}k_{2} \\
\left(\mathrm{~g} \mathrm{mg}^{-1} \mathrm{~h}^{-1}\right)\end{array}$ & $\begin{array}{c}h \\
\left(\mathrm{mg} \mathrm{g}^{-1} \mathrm{~min}^{-1}\right)\end{array}$ & $\begin{array}{l}t_{1 / 2} \\
(\mathrm{~h})\end{array}$ & $R^{2}$ \\
\hline ZIF-8 & 49.6 & 16.07 & 0.135 & 0.987 & 49.7 & 0.020 & 0.823 & 1.001 & 0.999 \\
\hline ZIF8-A15 & 99.9 & 36.65 & 0.159 & 0.991 & 101.3 & 0.009 & 1.529 & 1.097 & 0.998 \\
\hline ZIF8-A37 & 121.7 & 57.90 & 0.176 & 0.970 & 123.8 & 0.007 & 1.789 & 1.154 & 0.997 \\
\hline ZIF8-A61 & 174.8 & 75.04 & 0.191 & 0.988 & 178.9 & 0.004 & 2.134 & 1.397 & 0.999 \\
\hline
\end{tabular}

Table S2. Adsorption equilibrium constants of Langmuir and Freundlich isotherm equations for $I_{2}$ adsorption over the prepared adsorbents

\begin{tabular}{|c|c|c|c|c|c|c|}
\hline \multirow[b]{2}{*}{ Adsorbent } & \multicolumn{3}{|c|}{ Langmuir isotherm equation } & \multicolumn{3}{|c|}{ Freundlich isotherm equation } \\
\hline & $q_{m}\left(\mathrm{mg} \mathrm{g}^{-1}\right)$ & $K_{L}\left(\mathrm{~L} \mathrm{mg}^{-1}\right)$ & $R^{2}$ & $1 / n$ & $K_{F}\left(\mathrm{mg} \mathrm{g}^{-1}\right)$ & $R^{2}$ \\
\hline ZIF-8 & 131.8 & $0.70 \times 10^{-3}$ & 0.964 & 0.359 & 4.61 & 0.947 \\
\hline ZIF8-A15 & 574.7 & $0.34 \times 10^{-3}$ & 0.992 & 0.508 & 4.64 & 0.960 \\
\hline ZIF8-A37 & 961.5 & $0.25 \times 10^{-3}$ & 0.996 & 0.576 & 3.81 & 0.977 \\
\hline ZIF8-A61 & 1140.3 & $0.36 \times 10^{-3}$ & 0.997 & 0.530 & 7.62 & 0.971 \\
\hline
\end{tabular}


Table S3. The calculated atomic charge of ZIF-8 and ZIF8-A15 before and after I2 adsorption analyzed by the natural bond orbital method

\begin{tabular}{|c|c|c|c|c|}
\hline \multirow{2}{*}{ Phase } & \multirow{2}{*}{ Adsorbents } & \multirow{2}{*}{ Atom } & \multicolumn{2}{|c|}{ Calculated atomic charge } \\
\hline & & & before $I_{2}$ adsorption & after $I_{2}$ adsorption \\
\hline \multirow{6}{*}{$\begin{array}{l}\text { Liquid phase } \\
\text { (n-hexane) }\end{array}$} & ZIF-8 & $\mathrm{H}_{l}^{\alpha}$ & 0.197 & 0.201 \\
\hline & & $\mathrm{I}_{l}^{\alpha}$ & 0 & -0.266 \\
\hline & & $\mathrm{I}_{l}^{\alpha}$ & 0 & -0.284 \\
\hline & ZIF8-A15 & $\mathrm{H}_{l}^{\beta}$ & 0.207 & 0.223 \\
\hline & & $\mathrm{I}_{l}^{\beta}$ & 0 & -0.453 \\
\hline & & $\mathrm{I}_{l}^{\beta}$ & 0 & -0.442 \\
\hline \multirow{6}{*}{ Gas phase } & ZIF-8 & $\mathrm{H}_{g}^{\alpha}$ & 0.202 & 0.214 \\
\hline & & $\mathrm{I}_{g}^{\alpha}$ & 0 & -0.172 \\
\hline & & $\mathrm{I}_{g}^{\alpha}$ & 0 & -0.144 \\
\hline & ZIF8-A15 & $\mathrm{H}_{g}{ }^{\beta}$ & 0.200 & 0.233 \\
\hline & & $\mathrm{I}_{g}^{\beta}$ & 0 & -0.347 \\
\hline & & $\mathrm{I}_{g}^{\beta}$ & 0 & -0.272 \\
\hline
\end{tabular}




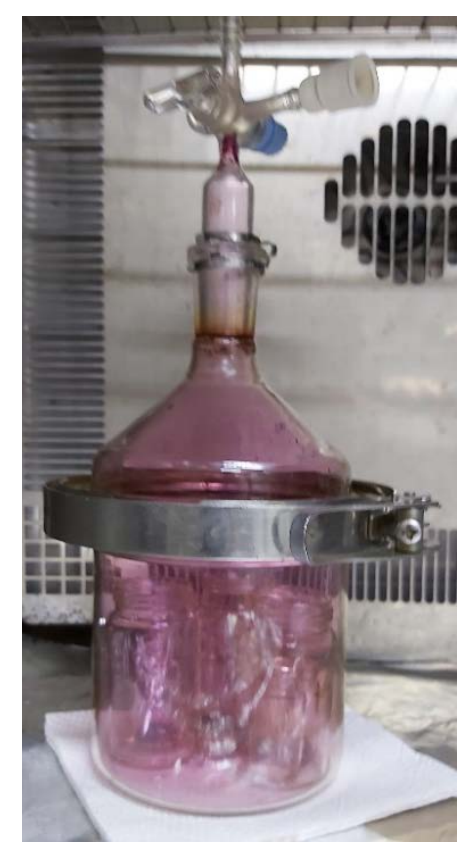

Scheme S1. Photograph of the apparatus used for vapor $\mathrm{I}_{2}$ adsorption.

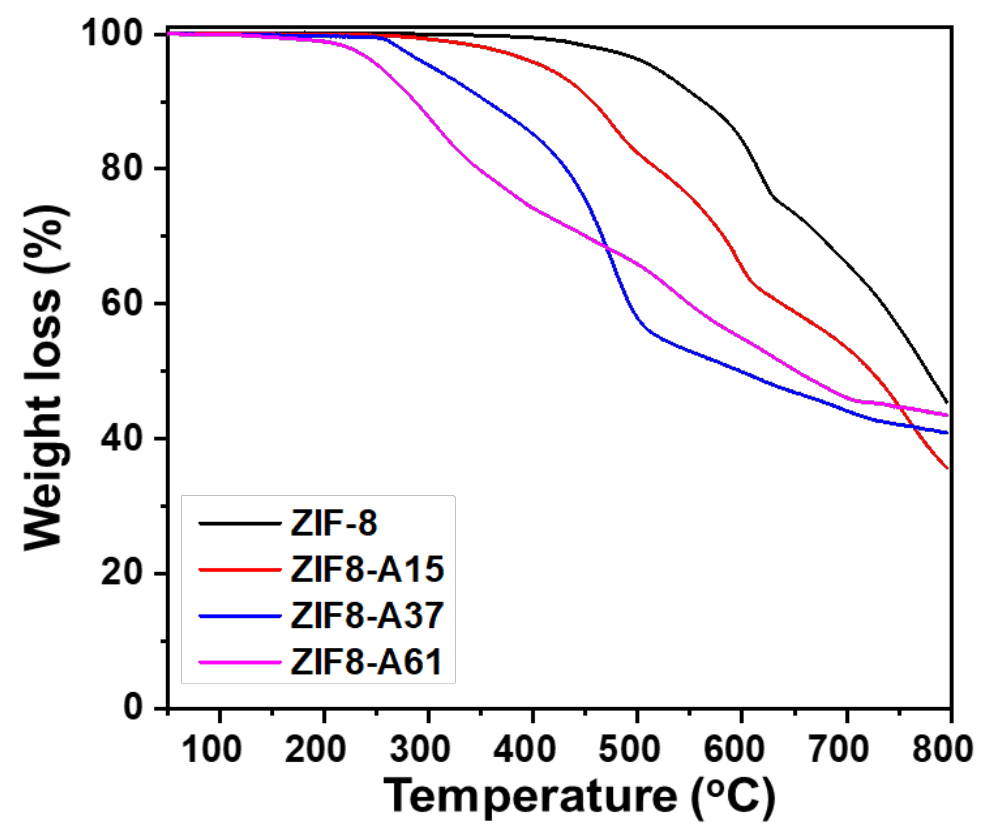

Figure S1. The TGA curve of the prepared materials. 


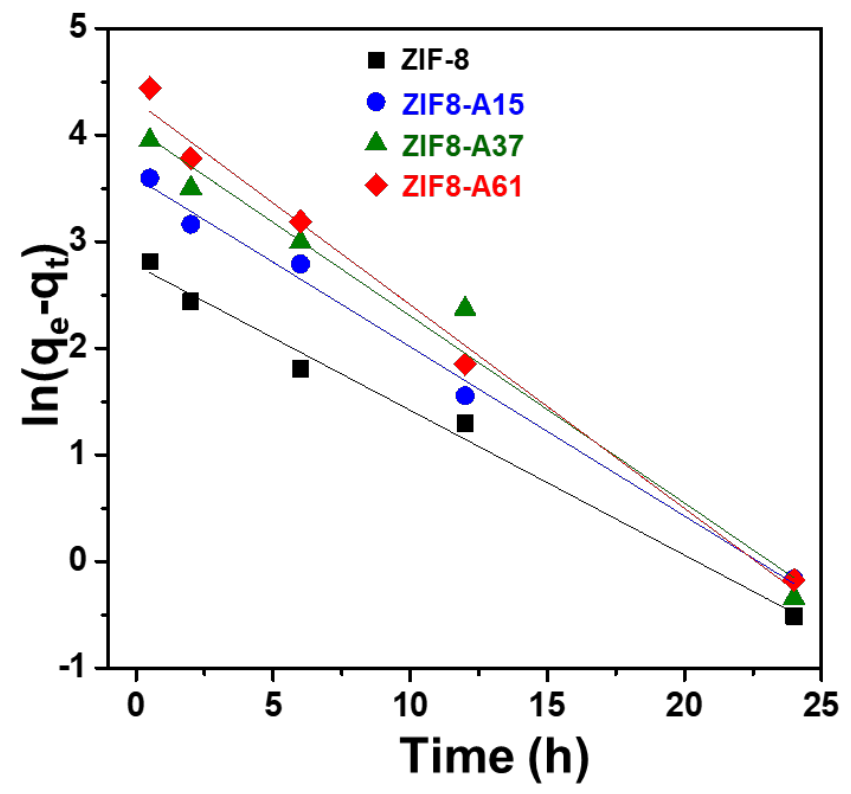

Figure S2. The pseudo-first-order kinetics model plots for $\mathrm{I}_{2}$ adsorption over ZIF-8 and ZIF8A materials.

(a)

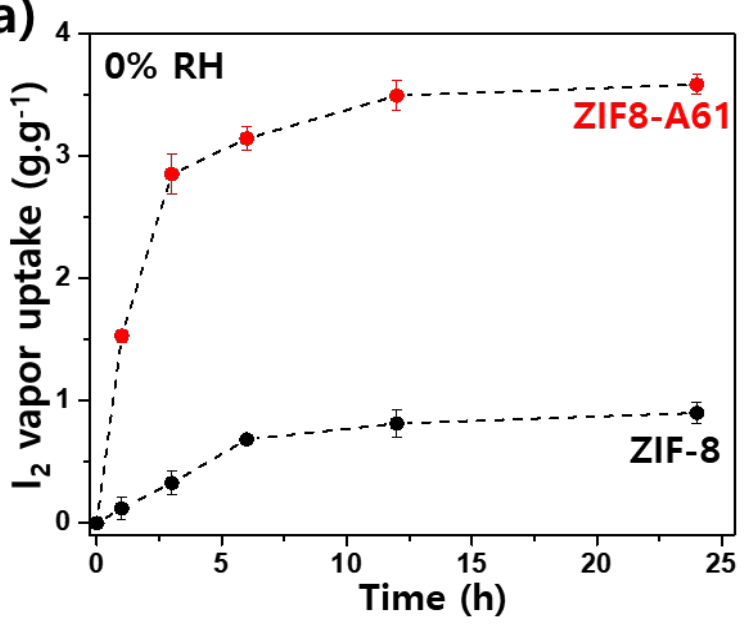

(b)

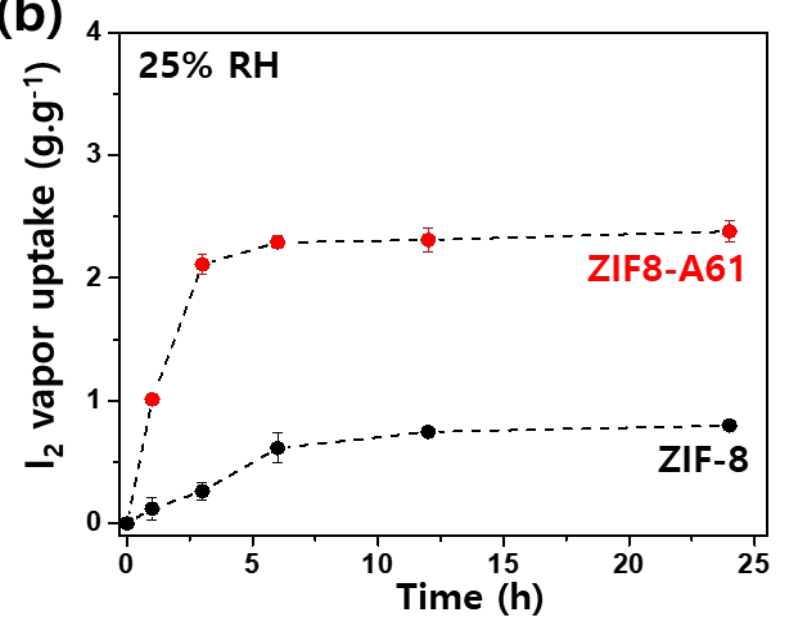

Figure S3. Adsorption curves of the ZIF-8 and ZIF8-A61 at $75{ }^{\circ} \mathrm{C}$ in saturated $\mathrm{I}_{2}$ vapor with (a) $0 \% \mathrm{RH}$ and (b) $25 \% \mathrm{RH}$. 

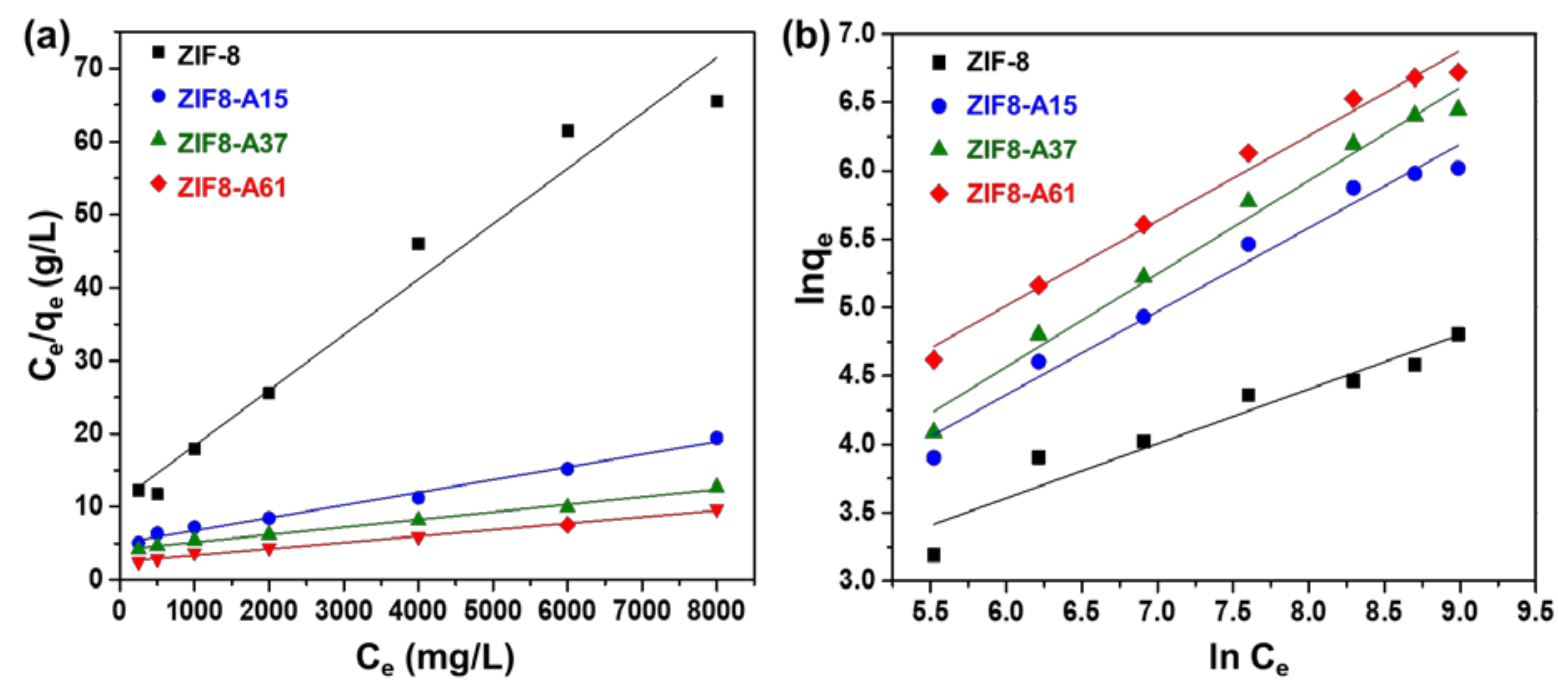

Figure S4. Langmuir isotherm (a) and Freundlich isotherm model (b) plots corresponding plots for $\mathrm{I}_{2}$ adsorption over the materials.

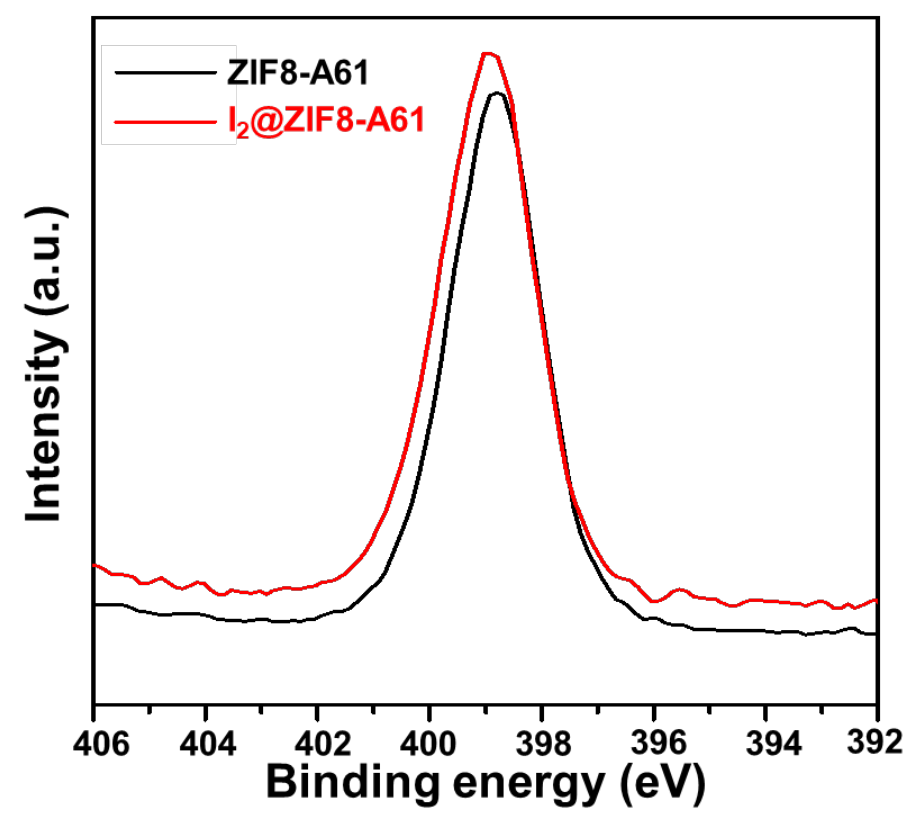

Figure S5. The high resolution XPS spectra of N 1s for ZIF8-A61 and I2@ZIF8-A61. 


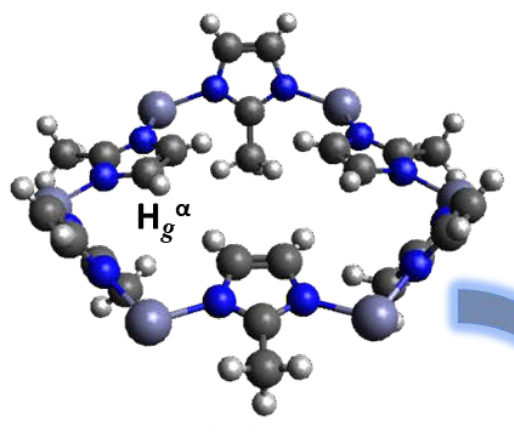

ZIF-8

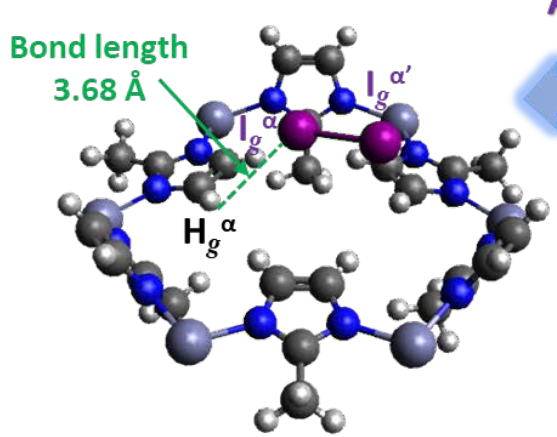

I2@ZIF-8

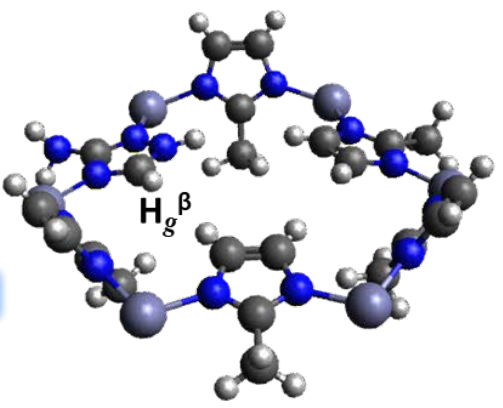

ZIF8-A15

ADSORPTION

Bond length

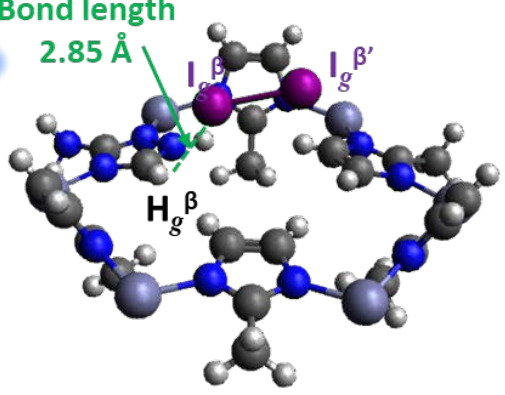

I $@ Z I F 8-A 15$

$\Delta G=-11.9 \mathrm{kcal} / \mathrm{mol}$

Figure S6. The DFT-simulated molecular $I_{2}$ arrangement with bond length of $I_{2} \cdots$ ZIF frameworks (ZIF-8 and ZIF8-A15) after $\mathrm{I}_{2}$ adsorption in gaseous phase and calculated the change of Gibbs free energies (Zn: blue gray, N: blue, C: gray, H: white, I: purple). 


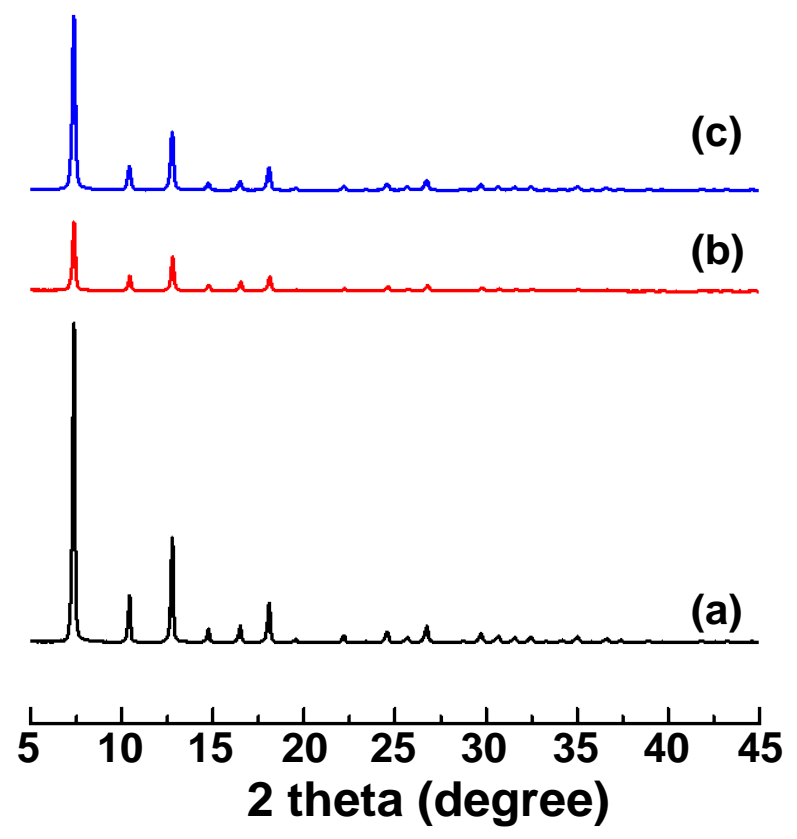

Figure S7. PXRD pattern of (a) ZIF-8, (b) I2 loaded ZIF-8 (I2@ZIF-8), and (c) I $\mathrm{I}_{2} @ Z I F-8$ releasing $\mathrm{I}_{2}$.

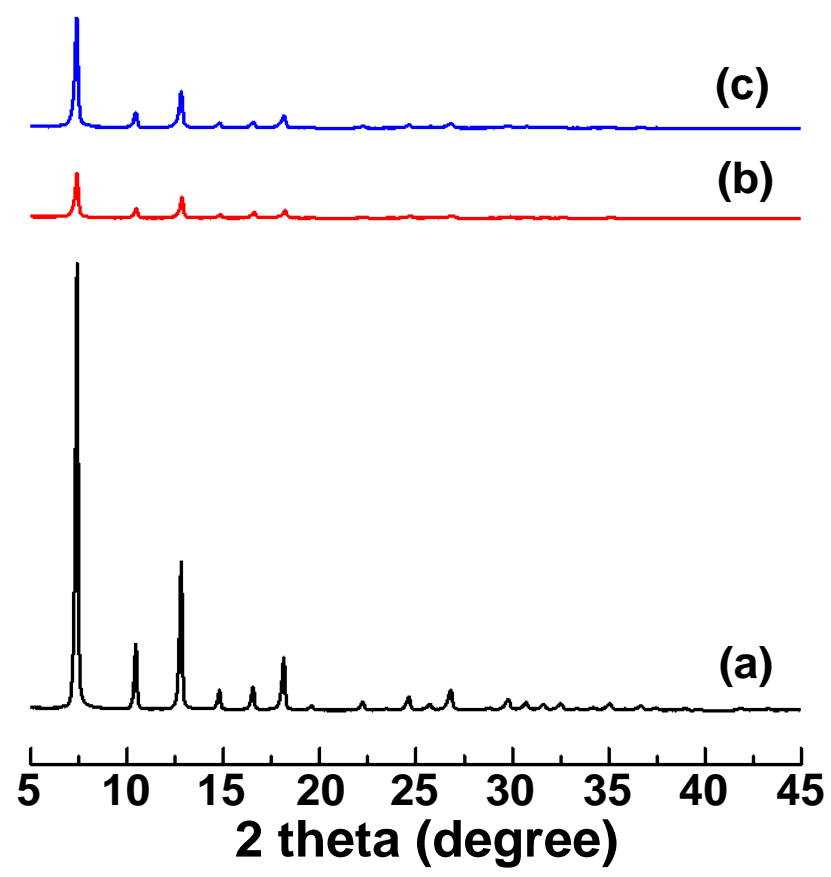

Figure S8. PXRD pattern of (a) ZIF8-A15, (b) I loaded ZIF8-A15 (I $\left.\mathrm{I}_{2} @ Z I F 8-A 15\right)$, and (c) I2@ZIF8-A15 releasing I2. 


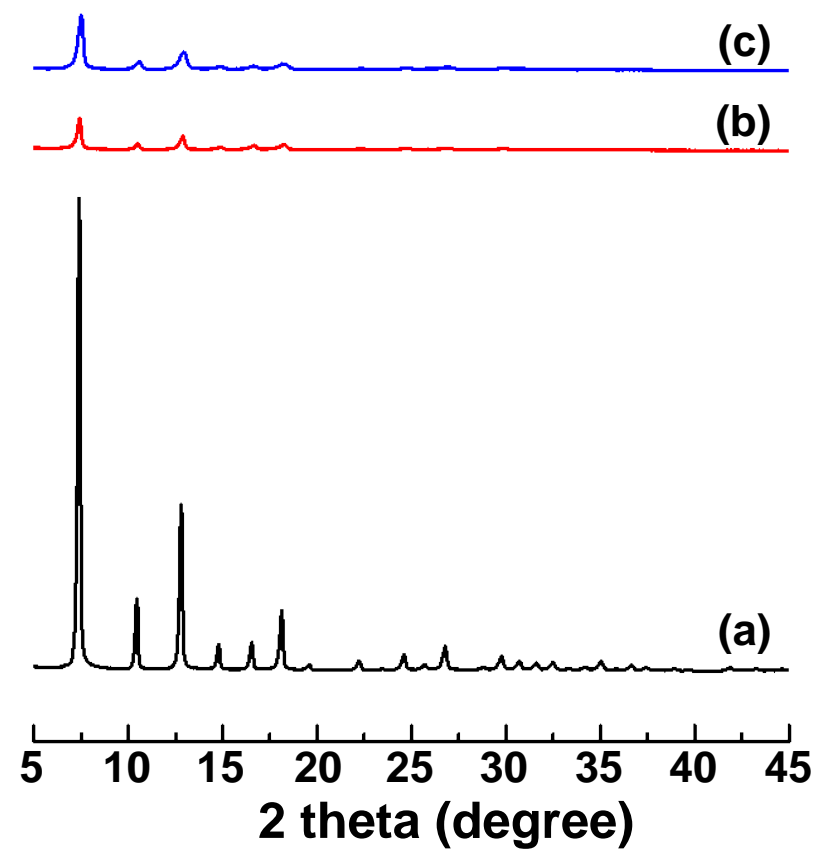

Figure S9. PXRD pattern of (a) ZIF8-A37, (b) $\mathrm{I}_{2}$ loaded ZIF8-A37 ( $\left.\mathrm{I}_{2} @ Z I F 8-A 37\right)$, and (c) I2@ZIF8-A37 releasing I2.

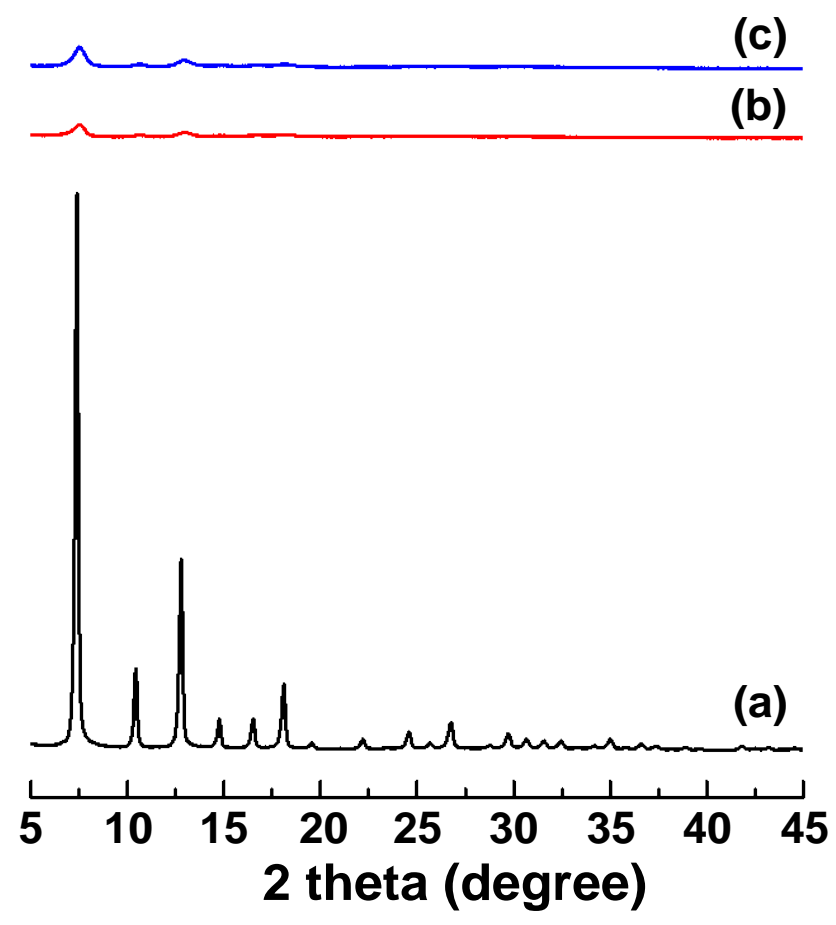

Figure S10. PXRD pattern of (a) ZIF8-A61, (b) I l loaded ZIF8-A61 (I $\left.\mathrm{I}_{2} @ Z I F 8-A 61\right)$, and (c) I2@ZIF8-A61 releasing I2. 


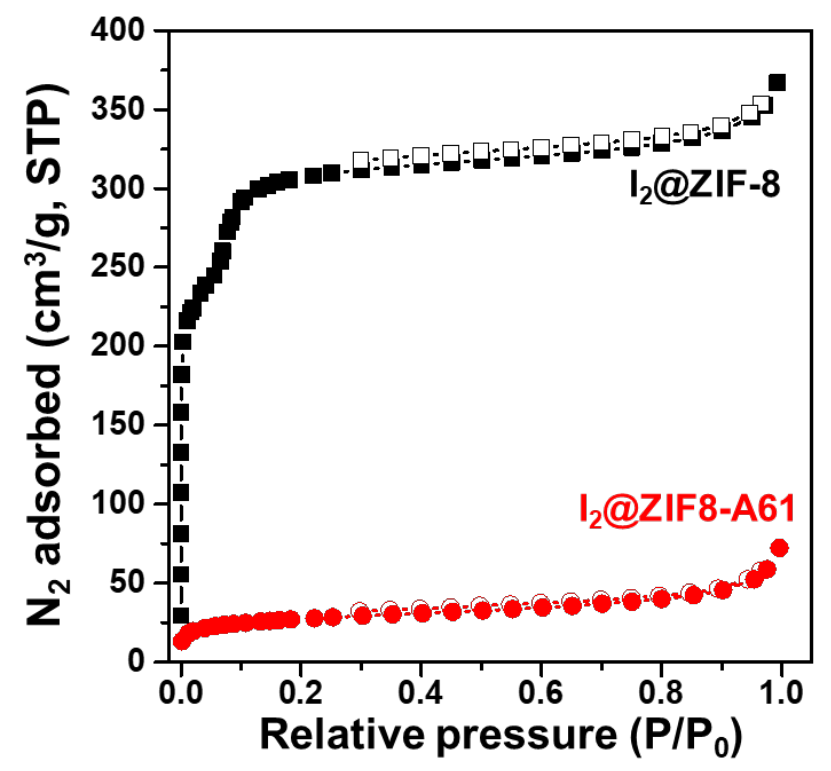

Figure S11. $\mathrm{N}_{2}$ adsorption-desorption isotherms of (a) ZIF-8 and (b) ZIF8-A61 before and after $\mathrm{I}_{2}$ adsorption.

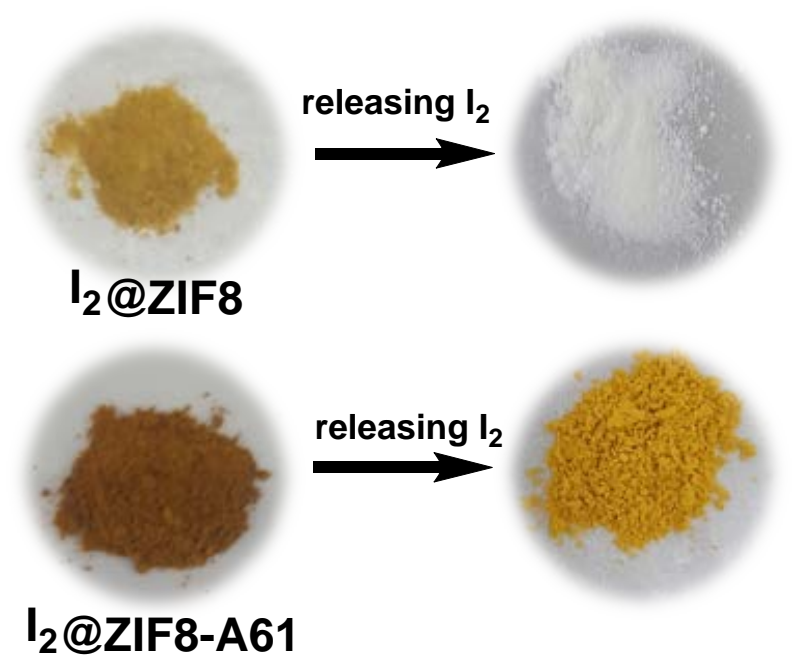

Figure S12. Photographs of ZIF-8 and ZIF8-A61 before and after releasing $\mathrm{I}_{2}$. 

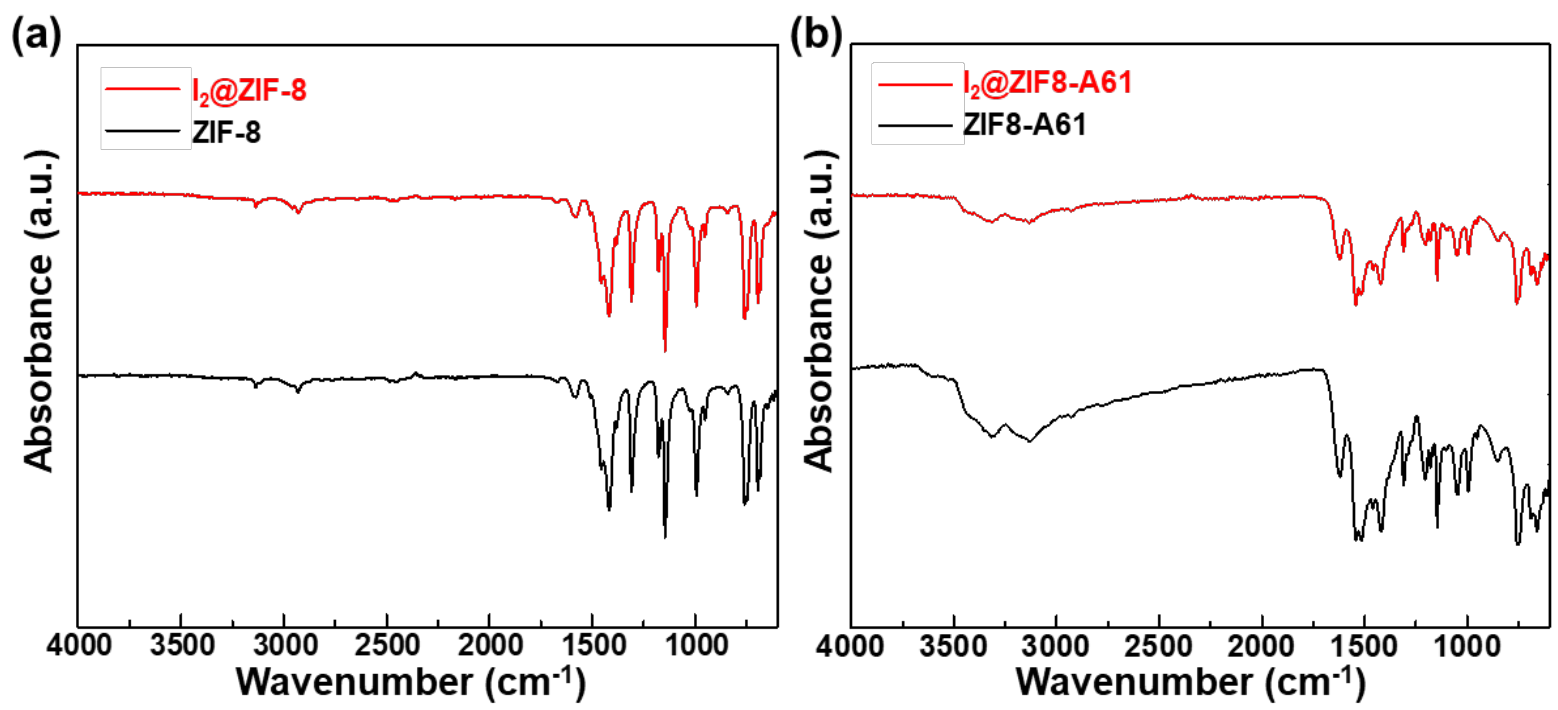

Figure S13. FT-IR spectra of (a) ZIF-8 and (b) ZIF8-A61 before and after I2 adsorption.

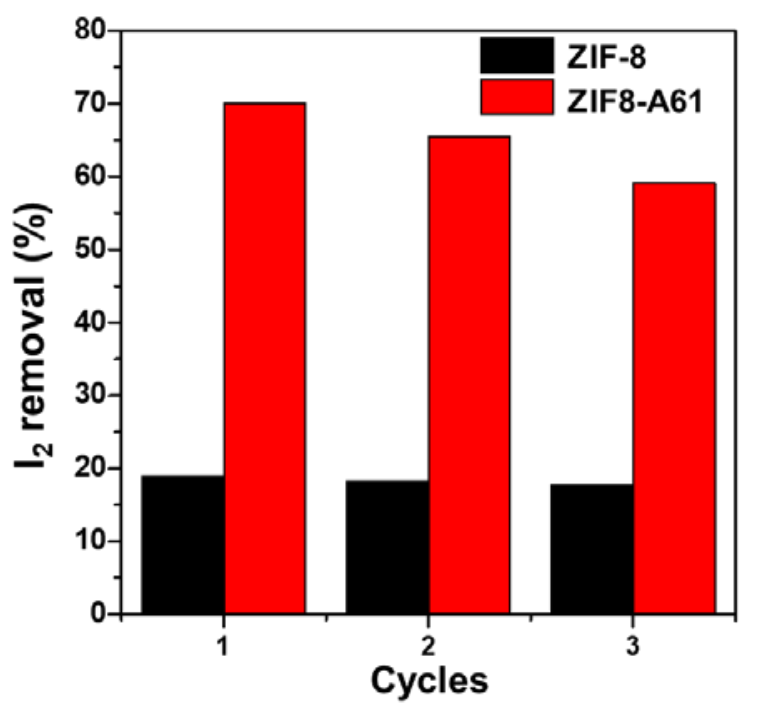

Figure S14. A reusability test of ZIF-8 and ZIF8-A61 for $\mathrm{I}_{2}$. 

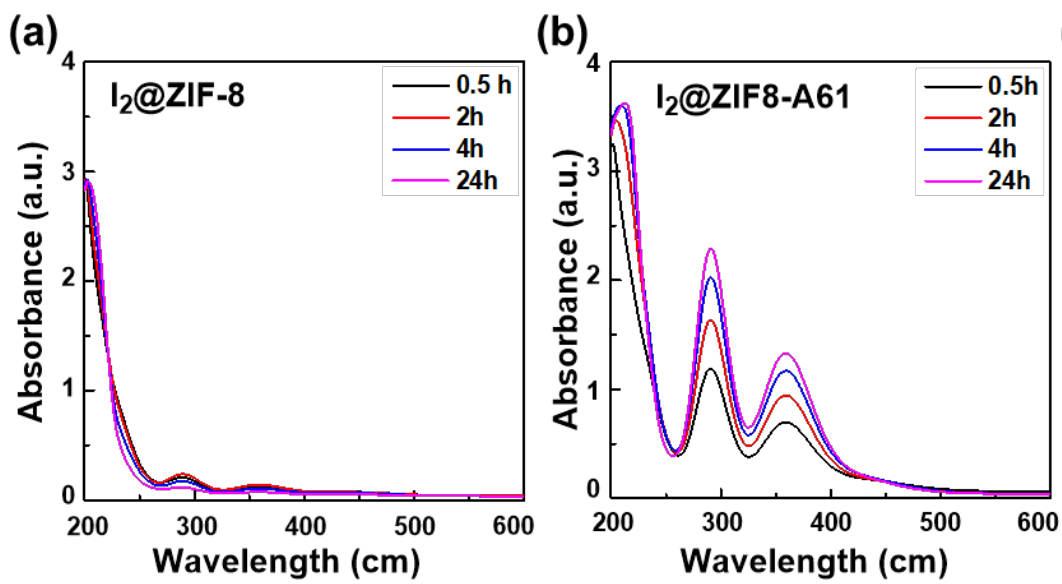

(c) oh $0.5 \mathrm{~h} 2 \mathrm{~h}: 4 \mathrm{~h} \quad 24 \mathrm{~h}$ I_@ZIF-8

Oh $0.5 h \quad 2 h \quad 4 h \geqslant 24 h$ T/1

Figure S15. UV-vis spectra of the $\mathrm{I}_{2}$ releasing process of (a) $\mathrm{I}_{2} @ Z$ ZIF-8 and (b) ZIF8-A61 immersed in ethanol for different contact time and (c) their corresponding photographs.
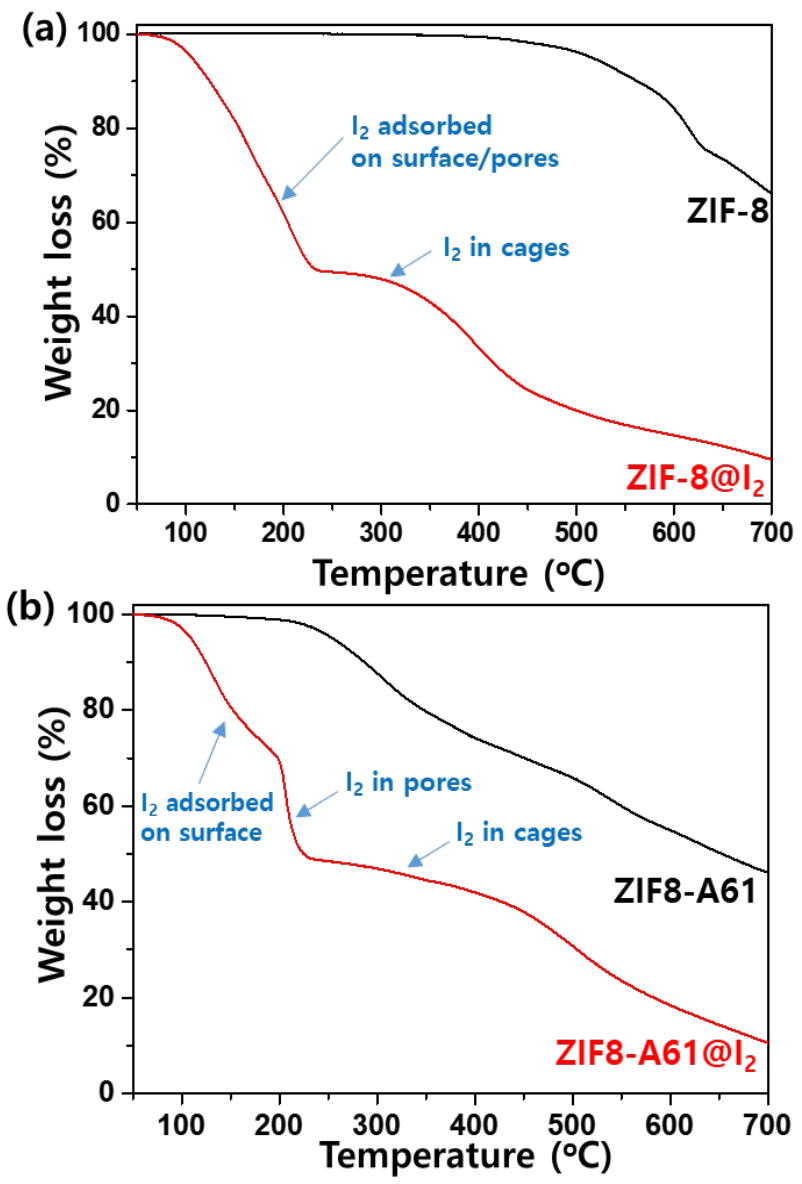

Figure S16. TGA curves of (a) ZIF-8 and ZIF-8@I I2 and (b) ZIF8-A61 and ZIF8-A61@I2. 


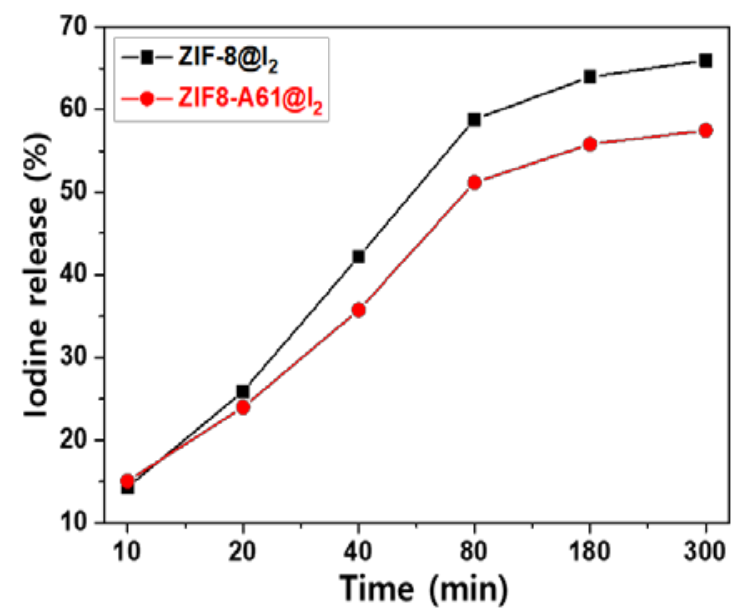

Figure S17. The kinetics of $\mathrm{I}_{2}$ release from ZIF-8@ $\mathrm{I}_{2}$ and ZIF8-A61@ $\mathrm{I}_{2}$.

\section{References}

[1] Cho, K. Y.; An, H.; Do, X. H.; Choi, K.; Yoon, H. G.; Jeong, H. -K.; Lee, J. S.; Baek, K. Y. J. Mater. Chem. A. 2018, 6, 18912.

[2] Wu, F. C.; Tseng, R. L.; Huang, S. C.; Juang, R. S. Characteristics of pseudo-second-order kinetic model for liquid-phase adsorption: A mini-review, Chem. Eng. J. 2009, 151, 1.

[3] Langmuir, I. The adsorption of gases on plane surfaces of glass, mica and platinum. J. Am. Chem. Soc. 1918, 40(9), 1361.

[4] Aytas, S.; Yurtlu, M.; Donat, R. Adsorption characteristic of U(VI) ion onto thermally activated bentonite, J. Hazard. Mater. 2009, 172(2-3), 667. 